\title{
Delusions in Individuals with Schizophrenia: Factor Structure, Clinical Correlates, and Putative Neurobiology
}

\author{
David Kimhy Ray Goetz Scott Yale Cheryl Corcoran Dolores Malaspina \\ Department of Psychiatry, Columbia University, and the Schizophrenia Research Unit, \\ Department of Medical Genetics, New York State Psychiatric Institute, New York, N.Y., USA
}

\section{Key Words}

Delusions · Schizophrenia • Factor analytic studies •

Neurobiology $\cdot$ Attribution

\begin{abstract}
Background: Delusions are a central feature of schizophrenia, yet our understanding of their neurobiology is limited. Attempt to link dimensions of psychopathology to putative neurobiological mechanisms depends on careful delineation of symptoms. Previous factor analytic studies of delusions in schizophrenia were limited by several methodological problems, including the use of patients medicated with antipsychotics, inclusion of nondelusion symptoms in the analyses, and/or inclusion of patients with psychotic disorders other than schizophrenia. These problems may have possibly biased the resulting factor structure and contributed to the inconclusive findings regarding the neurobiology of positive symptoms. Our goal is to examine the factor structure of delusions in antipsychotic-free individuals with diagnoses of schizophrenia/schizoaffective disorder. Sampling and Methods: We assessed 83 antipsychotic-free individuals with DSM-IV diagnoses of schizophrenia/ schizoaffective disorder. A principal component analysis was conducted on the delusions symptoms of the SAPS. Results: The principal component analysis resulted in three distinct and interpretable factors explaining $58.3 \%$
\end{abstract}

of the variance. The Delusions of Influence factor was comprised by delusions of being controlled, thought withdrawal, thought broadcasting, thought insertion, and mind reading. The Self-Significance Delusions factor was comprised by delusions of grandeur, reference, religious, and delusions of guilt/sin. The Delusions of Persecution factor was comprised solely by persecutory delusions. The three factors displayed distinct associations with hallucinations, bizarre behavior, attention, positive formal thought disorder, and avolition/apathy. Conclusions: The results indicate that delusions are best described by three distinct subtypes. The authors propose a novel model linking the three delusion subtypes, attributions to self/other, and putative neurobiological mechanisms. Implications for future research are discussed, as well as links to cognitive-behavioral conceptualizations of delusions.

Copyright $\odot 2005$ S. Karger AG, Basel

One of the major challenges of psychiatry is the attempt to link dimensions of psychopathology to putative neurobiological mechanisms. Delusions, as the 'basic characteristics of madness' [1], have attracted much theoretical interest, but surprisingly few experimental studies [2]. As a result, many of the fundamental questions about the phenomenology of delusions remain unanswered. To date, it is still unclear whether different types

\section{KARGER \\ Fax +4161306 1234 E-Mail karger@karger.ch} www.karger.com
(C) 2005 S. Karger AG, Basel 0254-4962/05/0386-0338\$22.00/0

Accessible online at:

www.karger.com/psp
David Kimhy, $\mathrm{PhD}$

Columbia University, Department of Psychiatry

Box \#2, 1051 Riverside Drive

New York, NY 10032 (USA)

Tel. +1 212543 6817, Fax +1 212543 6176, E-Mail dk553@columbia.edu 
of delusions reflect a unitary phenomenon with common neurobiological underpinnings. This lack of knowledge is of great consequence given the status of delusions as the most prevalent psychotic symptom among individuals with schizophrenia, and the evidence that different types of delusions respond differently to atypical antipsychotic medications [3].

A number of methodological problems have hindered the efforts to identify the neurobiology of delusions. One problem is associated with the scope of investigation - the great majority of studies searching for links between schizophrenia symptoms and underlying neurobiological mechanisms have employed global ratings of symptoms, such as the total positive or negative symptom scores. In such studies, ratings of delusions and hallucinations are typically aggregated under the rubric of positive or psychotic symptoms. Results from such studies have demonstrated that negative symptoms are often associated with both structural [4-8] and functional brain abnormalities [9-15]. In contrast, investigations of such links for global ratings of positive symptoms [14-16] have been inconclusive. This discrepancy could result if positive symptoms were more heterogeneous than negative symptoms $[17,18]$. Consequently, a number of investigators have argued that analysis of individual, item-level positive symptoms may yield more meaningful results than global symptom ratings [19-24].

A second problem is rooted in the instruments of assessment - the PANSS [25] and SAPS [26] are among the most widely used instruments designed to assess delusions and other positive symptoms. Historically, the factor structure of these instruments was derived from factor analytic studies that jointly considered delusions, other positive symptoms, negative symptoms, and other psychopathology items. However, since delusions may have a neurobiology that is distinct from other positive symptoms [27], the variance of the nondelusion symptoms in such analyses may have skewed the factor structure of delusions. Thirdly, the original factor structure of both instruments was developed using patients who were medicated. Different psychotic symptoms have different response rates to antipsychotic medications [3]. This may have further biased the factor structure. Furthermore, some factor analytic studies included subjects with psychotic disorders other than schizophrenia/schizoaffective disorders [17, 18]. Altogether, these methodological problems may have contributed to the inconclusive results regarding the neurobiology of delusions and positive symptoms.
The attempt to link delusions to putative neurobiological mechanisms depend on careful delineation of delusional symptoms. Such analysis could provide information about the individuality or overlap of subtypes of delusions, independent of other symptoms, which could serve as the foundation for identifying relevant neurobiological mechanisms, as well as distinct cognitive processes associated with each subtype. No study, to our knowledge, has assessed the factor structure of individual, item-level delusions separately from other symptoms (psychotic or others), let alone under antipsychotic-free status. We examined the factor structure of delusional phenomenology in antipsychotic-free individuals with schizophrenia by analyzing item-level delusions apart from other symptoms. We hypothesized that delusions will display a heterogeneous factor structure with clearly distinguishable factors.

\section{Method}

\section{Participants}

Participants were 83 individuals who successfully completed a 14-day antipsychotic-free period during their hospitalization at the Schizophrenia Research Unit at the New York State Psychiatric Institute. The study was approved by the Institutional Review Board of the New York State Psychiatric Institute, and after complete description of the study to the subjects, written informed consent was obtained from all participants. Inclusion criteria were age 18 years or older and a DSM-IV diagnosis of schizophrenia or schizoaffective disorder. Excluded were subjects who were acutely intoxicated or in withdrawal, subjects not fluent in English, and/or subjects unable to give informed consent. Table 1 displays the clinical characteristics of the sample.

\section{Measures}

Diagnosis. The Diagnostic Interview for Genetic Studies (DIGS) was administered to determine diagnosis. The DIGS is a structured diagnostic interview and review of medical records that is used to gather demographic, diagnostic, and course of illness information for the major affective, psychotic, and substance use DSM-IV axis I disorders. The DIGS has been shown to have good reliability in our group [28]. Diagnoses were based on the interviews, past records, and symptom ratings. The diagnosis was achieved by consensus among clinical and research staff.

Symptom Ratings. The Scales for Assessment of Positive and Negative Symptoms [26] were used to assess delusional, positive, and negative symptoms. The SAPS/SANS include 34 item-level positive symptoms (including 12 types of delusions) and 25 itemlevel negative symptoms. Symptoms were assessed during hospitalization following the completion of at least 14 antipsychotic-free days. Delusions of Jealousy (item No. 9 on the SAPS) were assessed but excluded from the data analysis due to the fact that this item does not apply to all subjects (subjects must be married or in a relationship in order to be able to respond to this item). Additionally, this item was the least endorsed item by participants. 
Table 1. Demographic and clinical characteristics of the sample $(\mathrm{n}=83)$

\begin{tabular}{|c|c|c|c|}
\hline & Mean & SD & Range \\
\hline \multicolumn{4}{|l|}{ Diagnosis } \\
\hline Schizophrenia & $62(75 \%)$ & - & - \\
\hline Schizoaffective & $21(25 \%)$ & - & - \\
\hline \multicolumn{4}{|l|}{ Sex } \\
\hline Male & $50(60 \%)$ & - & - \\
\hline Female & $33(40 \%)$ & - & - \\
\hline \multicolumn{4}{|l|}{ Ethnicity } \\
\hline White & $39(47 \%)$ & - & - \\
\hline African-American & $21(25 \%)$ & - & - \\
\hline Hispanic & $17(21 \%)$ & - & - \\
\hline Asian & $2(2 \%)$ & - & - \\
\hline Multiethnic & $4(5 \%)$ & - & - \\
\hline Age & 35.27 & 10.48 & $18-60$ \\
\hline Education & 12.41 & 2.44 & $6-16$ \\
\hline Duration of illness & 12.28 & 10.60 & $0-37$ \\
\hline Age at onset of psychotic symptoms & 22.41 & 7.67 & $9-42$ \\
\hline Number of hospitalizations & 6.41 & 6.36 & $1-26$ \\
\hline GAS score & 31.56 & 7.50 & $18-50$ \\
\hline \multicolumn{4}{|l|}{ Course of illness $(n=49)$} \\
\hline Episodic shift/mild deterioration & $6(12 \%)$ & - & - \\
\hline Moderate deterioration & $25(51 \%)$ & - & - \\
\hline Severe deterioration/stable poor & $18(37 \%)$ & - & - \\
\hline $\begin{array}{l}\text { Number of subjects with delusions } \\
\quad \text { (SAPS rating } \geq 2 \text { ) }\end{array}$ & $58(70 \%)$ & - & - \\
\hline \multicolumn{4}{|l|}{ Delusions } \\
\hline Persecutory & 1.31 & 1.58 & $0-5$ \\
\hline Guilt/sin & 0.40 & 0.92 & $0-4$ \\
\hline Grandiose & 0.82 & 1.39 & $0-5$ \\
\hline Religious & 0.58 & 1.15 & $0-5$ \\
\hline Somatic & 0.93 & 1.50 & $0-5$ \\
\hline Reference & 1.00 & 1.61 & $0-5$ \\
\hline Being controlled & 0.71 & 1.42 & $0-5$ \\
\hline Mind reading & 0.89 & 1.49 & $0-5$ \\
\hline Broadcasting & 0.71 & 1.38 & $0-5$ \\
\hline Thought insertion & 0.53 & 1.22 & $0-5$ \\
\hline Thought withdrawal & 0.73 & 1.41 & $0-5$ \\
\hline Global ratings of delusions & 2.42 & 1.73 & $0-5$ \\
\hline
\end{tabular}

GAS = Global assessment scale; episodic shift/mild deterioration $=$ episodes of illness are interspersed by periods of health with mild or no deterioration in functioning; moderate deterioration = occasional resolution of symptoms may occur, but overall the course is downhill culminating in moderate degree of social and occupational functioning; severe deterioration/stable poor $=$ the subject's illness has become chronic resulting in social impairment and inability to maintain employment. Delusions ratings based on the SAPS: 0 (none) to 5 (severe).
Table 2. Principal component analysis for the 11 delusion itemlevel symptoms

\begin{tabular}{lrrr}
\hline & \multicolumn{3}{l}{ Delusions of } \\
\cline { 2 - 4 } & Influence & Self-Significance & Persecution \\
\hline Variance extracted, \% & 26.09 & 20.85 & 11.39 \\
Eigen values & 2.87 & 2.29 & 1.25 \\
\hline Being controlled & $\mathbf{0 . 7 6}$ & -0.16 & -0.06 \\
Thought withdrawal & $\mathbf{0 . 7 2}$ & 0.33 & 0.12 \\
Thought broadcasting & $\mathbf{0 . 6 3}$ & 0.45 & -0.08 \\
Thought insertion & $\mathbf{0 . 6 3}$ & 0.15 & 0.29 \\
Mind reading & $\mathbf{0 . 5 6}$ & 0.45 & 0.29 \\
Grandiose & 0.12 & $\mathbf{0 . 7 3}$ & 0.15 \\
Guilt/sin & -0.07 & $\mathbf{0 . 6 1}$ & 0.12 \\
Religious & 0.46 & $\mathbf{0 . 6 1}$ & -0.08 \\
Reference & 0.42 & $\mathbf{0 . 5 6}$ & -0.24 \\
Persecutory & 0.08 & 0.02 & $\mathbf{0 . 8 9}$ \\
Somatic & 0.48 & 0.17 & 0.40 \\
\hline
\end{tabular}

Bold type indicates variables loading on a particular factor.

\section{Procedures}

The diagnosis (DIGS) and symptom (SAPS/SANS) measures were administered by experienced masters' degree level clinicians. As part of an ongoing research protocol on the unit, subjects completed 4 weeks on stable antipsychotic doses, upon which the antipsychotics were slowly tapered until achievement of an antipsychotic-free status. The assessment of symptoms using the SAPS/ SANS was completed following at least 14 days of being antipsychotic-free. Antipsychotics were either typical or atypical, depending on the protocol being conducted when the patient was studied. Lorazepam (1-2 mg, i.m. or p.o.) was available for severe agitation, anxiety, or insomnia during any treatment condition.

\section{Results}

Principal component analysis using varimax rotation with Kaiser normalization resulted in three distinct and interpretable factors explaining $58.3 \%$ of the variance. The Kaiser-Meyer-Olken sampling adequacy measure was 0.78 , indicating that the data were adequate for factor analysis. Table 2 presents the principal-component structure after the components were rotated. The number of factors extracted was based on the Eigen value $\geq 1$ criterion. Symptoms were assigned to factors based on their highest level of loading. The results of the factor extraction suggest three distinct and interpretable factors. Factor 1 (Delusions of Influence) is comprised of delusions of being controlled, thought withdrawal, thought broadcasting, thought insertion, and mind reading. This factor 
Table 3. Correlations between delusion factors and total rating of symptoms

\begin{tabular}{lccc}
\hline & \multicolumn{3}{c}{ Delusions of } \\
\cline { 2 - 4 } & $\begin{array}{l}\text { Influence } \\
\text { Self- } \\
\text { Significance }\end{array}$ \\
\hline Affective flattening & -0.07 & -0.16 & -0.03 \\
Alogia & 0.02 & 0.18 & -0.11 \\
Avolition/apathy & $\mathbf{0 . 3 7 * *}$ & $\mathbf{0 . 3 1 * *}$ & -0.04 \\
Anhedonia/asociality & -0.09 & 0.03 & 0.12 \\
Attention & $0.27 *$ & 0.12 & -0.11 \\
Hallucinations & $\mathbf{0 . 4 6 * *}$ & -0.11 & 0.20 \\
Bizarre behavior & 0.08 & $\mathbf{0 . 4 4} * *$ & 0.05 \\
Positive formal thought disorder & -0.06 & $0.27 *$ & 0.11 \\
\hline
\end{tabular}

* Significance at $0.05 ; * *$ significance at 0.01 . Bold type indicates significant correlations after Bonferroni correction.

partially corresponds with Schneiderian 'first-rank' symptoms. Conversely, items in this factor also correspond with what has been termed in the German literature as Ichstörungen (ego-disturbance), which is considered by some authors as an experience that is distinct from delusions. Factor 2 (Delusions of Self-Significance) is comprised of delusions of grandeur, reference, religious, and delusions of guilt/sin. Factor 3 (Delusions of Persecution) is comprised of a single item - persecutory delusions. None of the item-level delusions loaded heavily $(\geq 50)$ on more than one factor. The only item-level delusion not loading on any of the three factors was somatic delusions. Post-hoc analysis revealed that they were related primarily to the presence of somatic hallucinations (partial $r=$ $0.642, \mathrm{p}<0.001$, controlling for all other types of hallucinations).

To assess the relationship of the extracted delusion factors with other clinical phenomena, we evaluated their correlations with other SAPS/SANS symptom dimensions (table 3). Delusions of Influence were significantly correlated with hallucinations, avolition/apathy, and poor attention. Increases in Self-Significance Delusions were significantly related to exacerbation of bizarre behavior, avolition/apathy, and positive formal thought disorder. Delusions of Persecution were not associated with other symptom categories. However, a post-hoc analysis revealed correlations with visual hallucinations (partial $r=-0.345, p=0.002$ ), controlling for other types of hallucinations.

While most factor analytic studies of symptoms in schizophrenia have used varimax rotation, some re- searchers [18] have argued for using an oblique rotation procedure (OBLIMIN) stating that it is more appropriate because it is more in line with the clinical sense that individuals present symptoms from various dimensions concurrently. To assess this argument, a principal component analysis using OBLIMIN rotation with Kaiser normalization was executed using the same data and the Eigen value $\geq 1$ criterion. This analysis also produced three distinct factors that explained $58.3 \%$ of the variance, with factor structure quite similar to the one obtained using the varimax rotation.

\section{Discussion}

The findings support our hypothesis that delusions are heterogeneous clinical phenomena. We found that delusions are best described by three distinct subtypes - Delusions of Influence, Self-Significance, and Persecution. Overall, the three extracted factors explained a substantial portion of the variance, as well as demonstrated a clean structure with most item-level delusions loading heavily on only one factor. Our results may be particularly significant and unique given the antipsychotic-free status of the patients. The assessment of symptoms in antipsychotic-free patients mitigates against potential confounding effects that arise due to certain subtypes of delusions responsive differentially to antipsychotic treatments [3].

Our results invite speculations about putative neurobiological mechanisms associated with each subtype of delusions. In particular, the anterior cingulate (AC; Brodmann's Area 24) and the medial prefrontal cortex (MPFC; Brodmann's Area 32) appear to play an important role in delusions. These regions are often joined in imaging studies, as the anatomical border between them is not rigid. The ventral part of the AC occupies most of the arc around the genu of the corpus callosum, and is cytoarchitecturally bound by the MPFC, also known as the anterior paracingulate cortex. The AC/MPFC is among the brain regions with the highest baseline metabolic activity at rest, with consistent decreases in activity during specific goaldirected behavior [29, 30]. Kelley et al. [31] found significant differences in AC/MPFC activation between judgments of self, others, and control tasks among healthy controls. Judgments of others and control tasks were accompanied by significant reduction in AC/MPFC hemodynamic activity. In contrast, judgments of self were associated with only minimal reductions from baseline. 
From a psychological perspective, delusions represent attempts by the individual to make sense of personal experiences by attributing causes to internal and/or external events. While copious in content and form, delusions can be divided into three subgroups based on types of attribution: (1) attributions to one's own psychological self; (2) attributions to other people or external objects, and/or (3) attributions to one's own physical self. We found SelfSignificance delusions to be associated with disorganized behavior and positive formal thought disorder. These findings are consistent with Sabri et al. [27] who reported that grandiosity and formal thought disorder were strongly associated with hyperperfusion in the AC/MPFC and frontal regions. Thus, the overactivation of the AC/MPFC that was found to be associated with grandiosity [27] and Delusions of Self-Significance, may reflect an excessive attribution of experiences to the self that is at the core of these symptoms. In contrast, persecutory delusions have been found to be correlated with hypoperfusion in the $\mathrm{AC} / \mathrm{MPFC}$ [27]. Thus, the reduction of AC/MPFC $\mathrm{rCBF}$ linked with persecutory delusions is associated with the excessive attributions to other people, and is consistent with the external attributional bias that is common among individuals with persecutory delusions. Blackwood et al. [32] reported significantly lower AC/MPFC activation during a task determining self-relevance among individuals with paranoid delusions compared with controls. Regarding Delusions of Influence, Sabri et al. [27] used the PANSS, which divides delusions into persecutory, grandiose, and other types. Since this delineation of delusions is different from our factor structure, it is difficult to draw conclusions about the relationship between Delusions of Influence and AC/MPFC activation. However, this factor contains delusions that often focus on attribution of internal experiences to external sources. Thus, it seems likely that such attributions may involve AC/MPFC hypoperfusion. This view is not exclusive to the model put forward by Gallagher and Frith [33], who proposed that delusions of passivity experience ('alien control') are rooted in sensory integration abnormalities in the superior temporal sulcus. Such abnormalities may be a subcategory of persecutory delusions that involves erroneous integration of physiological experiences, coupled with attribution of such experiences to malevolent external causes.

To date, attempts to link symptoms to neurobiology has focused primarily on use of global dimensions of symptoms (i.e., positive symptoms). However, given our findings and recent evidence that delusions are neurobiologically unique from other positive symptoms [27], it is not surprising that such attempts resulted in inconclusive findings. The use of global constructs, while conceptually and economically attractive, results in loss of associations with other clinical phenomena. In fact, the pursuit of such links using combined or aggregate scores of delusions and hallucinations may be akin to fishing for guppies with a net designed to catch tunas. Conversely, the use of itemlevel symptoms produces more specific characterization of clinical phenomena. However, such focus may blur the view of the global construct of schizophrenia.

One solution to this ongoing debate is to find an intermediate level of representation of clinical phenomena that preserves clinical details, without being lost in them. Peralta and Cuesta [34] suggested a hierarchical system of symptom classification that will facilitate the search for the most appropriate level of linkage of symptoms with neurobiological mechanisms. Accordingly, item-level symptoms (i.e., delusions of thought withdrawal) will be placed at the bottom of the hierarchy, followed by a more general category of symptoms (i.e., Delusions of Influence), followed by broader clinical constructs (i.e., positive symptoms). The conventional categorization of delusions based on content (e.g. religious, grandiose, etc.) hinders the ability to find links to the underlying neurobiological process. In contrast, a model that focuses on process (neurobiological and/or psychological) may allow for more precise ascertainment of the underlying neurobiological mechanisms associated with specific symptoms (i.e., delusions of thought withdrawal). Such a model would also allow to find their commonalities and differences with higher-order symptom categories (i.e., Delusions of Influence), as well as possible broader clinical constructs (i.e., positive symptoms). Our results fit well with this model of hierarchical symptom classification.

To date, only two studies have analyzed symptom structures using item-level symptoms, and their results partially correspond with the factor structure obtained in the present study. The factor analysis of all fifty SAPS/ SANS items of Toomey et al. [17] produced nine factors. The individual item-level delusion symptoms loaded on three factors - Bizarre Delusions (consisting of delusions of thought insertion, thought withdrawal, being controlled, thought broadcasting, and delusions of mind reading), Disorganization (that included grandiose delusions among other nondelusion symptoms), and Nonbizarre delusions (consisting of delusions of persecution, reference, jealousy, and sin/guilt). Similarly, Peralta and Cuesta [18] conducted a factor analysis of all fifty SAPS/ SANS items that produced twelve factors. The individ- 
ual item-level delusion symptoms loaded on five factors - delusions of being controlled, mind reading, thought broadcasting, thought insertion, and delusions of thought withdrawal loaded on one factor. Grandiose, religious, and guilt/sin delusions loaded on a second factor. Delusions of persecution and reference loaded on a third factor. Somatic delusions, which did not load heavily on any factor in the present study, loaded on a fourth factor along with visual, olfactory, and somatic hallucinations. Finally, delusions of jealousy, which were excluded from the present study data analysis, loaded separately on a fifth factor. The discrepancies between these factor structures and our results could be attributed to these studies including in their analysis individuals with psychotic disorders other than schizophrenia, the inclusion of nondelusion symptoms in the analyses, as well as the inclusion of subjects medicated with antipsychotics.

Comments on our methodology may be particularly pertinent. While some researchers [18] have argued for using OBLIMIN in analysis of factors, we adopted the varimax procedure for a number of reasons. First, the two rotation techniques appear to produce relatively small differences in our data, as well as the data presented by Peralta and Cuesta [18]. Secondly, the purpose of the present investigation was to identify distinct categories of symptoms that will elucidate the complex clinical picture of delusions in schizophrenia. Thus, a statistical technique that accentuates differences between factors appeared more suitable. Thirdly, results from previous studies [27] are more consistent with the factor structure extracted using the varimax technique, as compared with the one obtained by the oblique method.

One limitation of the present study is the relatively modest sample size $(n=83)$. As a rule of thumb, a reliable factor structure requires a minimum patient/variable ratio of $10 / 1$. In this study, this ratio was $7.6 / 1$. While a greater sample size would have been preferable, the difficulties of accessing and studying antipsychotic-free individuals with schizophrenia make such requirement extremely challenging. However, given the sample size and possibility that the resulting factor structure may not be reliable, these results need to be replicated with a larger sample of antipsychotic-free individuals with schizophrenia. Similarly, the relatively modest sample size prevented drawing conclusions about possible differences in delusions between individuals with schizophrenia and schizoaffective disorders.

Our belief is that the delineation of precise domains of delusions will facilitate the discovery of links between symptoms, specific neurobiological mechanisms, as well as associated cognitive processes. In the absence of such precise domains, the links between symptoms, neurobiology, and cognition will remain clouded by influences of other clinical phenomena. Thus, the results of this study have implications for future research. Primarily, researchers should study subtypes of delusions rather than consider delusions as a homogeneous clinical category. Secondly, the current factor structure should be replicated with other samples. Thirdly, researchers should investigate the link between these subtypes and putative neurobiological mechanisms. A forth line of research should focus on the temporal stability of the delusion factors and their relationship to specific stages of illness. Finally, recent reports indicate that delusions are associated with a cognitive style that is characterized by 'jumping to conclusions', an externalizing attributional bias, and poor theory of mind [35]. However, it is not clear whether these cognitive processes apply to all types of delusions or just some of them. Investigations of the cognitive, affective and neurobiological correlates of specific delusion subtypes may lead to the development of more refined cognitive theories of delusions and better targeted cognitivebehavioral interventions that will address specific phenomena associated with each subtype of delusions. 


\section{References}

1 Jaspers K: General Psychopathology. Man- 13 Molina Rodriguez V, Montz Andree R, Perez chester, Manchester University Press, 1963.

2 Roberts G: The origins of delusion. Br J Psychiatry 1992;161:298-308.

3 Breier A, Berg PH: The psychosis of schizophrenia: Prevalence, response to atypical antipsychotics, and prediction of outcome. Biol Psychiatry 1999;46:361-364.

-4 Gur RE, Mozley PD, Shtasel DL, Cannon TD, Gallacher F, Turetsky B, Grossman R, Gur $\mathrm{RC}$ : Clinical subtypes of schizophrenia: Differences in brain and CSF volume. Am J Psychiatry 1994; $151: 343-350$.

5 Wible CG, Anderson J, Shenton ME, Kricun A, Hirayasu Y, Tanaka S, Levitt JJ, O’Donnell BF, Kikinis R, Jolesz FA, McCarley RW: Prefrontal cortex, negative symptoms, and schizophrenia: An MRI study. Psychiatry Res 2001; 108:65-78

-6 Anderson JE, Wible CG, McCarley RW, Jakab M, Kasai K, Shenton ME: An MRI study of temporal lobe abnormalities and negative symptoms in chronic schizophrenia. Schizophr Res 2002;58:123-134.

-7 Ho BC, Andreasen NC, Nopoulos P, Arndt S, Magnotta V, Flaum M: Progressive structural brain abnormalities and their relationship to clinical outcome: A longitudinal magnetic resonance imaging study early in schizophrenia. Arch Gen Psychiatry 2003;60:585-594.

-8 Szeszko PR, Gunning-Dixon F, Ashtari M, Snyder PJ, Lieberman JA, Bilder RM: Reversed cerebellar asymmetry in men with firstepisode schizophrenia. Biol Psychiatry 2003; 53:450-459.

-9 Brodie JD, Christman DR, Corona JF, Fowler JS, Gomez-Mont F, Jaeger J, Micheels PA, Rotrosen J, Russell JA, Volkow ND: Patterns of metabolic activity in the treatment of schizophrenia. Ann Neurol 1984;15(suppl):S166S169.

10 Lewis SW, Ford RA, Syed GM, Reveley AM, Toone BK: A controlled study of $99 \mathrm{mTc}-\mathrm{HM}-$ PAO single-photon emission imaging in chronic schizophrenia. Psychol Med 1992;22:2735.

11 Wolkin A, Sanfilipo M, Wolf AP, Angrist B, Brodie JD, Rotrosen J: Negative symptoms and hypofrontality in chronic schizophrenia. Arch Gen Psychiatry 1992;49:959-965.

-12 Schroder J, Buchsbaum MS, Siegel BV, Geider FJ, Lohr J, Tang C, Wu J, Potkin SG: Cerebral metabolic activity correlates of subsyndromes in chronic schizophrenia. Schizophr Res 1996; 19:41-53.
Castejon MJ, Gutierrez Labrador R, Ferre Navarrete F, Carreas Delgado JL, Rubia Vila FJ: Cerebral perfusion correlates of negative symptomatology and parkinsonism in a sample of treatment-refractory schizophrenics: An exploratory 99mTc-HMPAO SPET study. Schizophr Res 1997;25:11-20.

14 Min SK, An SK, Jon DI, Lee JD: Positive and negative symptoms and regional cerebral perfusion in antipsychotic-naive schizophrenic patients: A high-resolution SPECT study. Psy-

-15 Potkin SG, Alva G, Fleming K, Anand R, Keator D, Carreon D, Doo M, Jin Y, Wu JC, Fallon JH: A PET study of the pathophysiology of negative symptoms in schizophrenia. Am J Psychiatry 2002;159:227-237.

-16 Arango C, Breier A, McMahon R, Carpenter WT, Buchanan RW: The relationship of clozapine and haloperidol treatment response to prefrontal, hippocampal, and caudate brain volumes. Am J Psychiatry 2003;160:14211427.

17 Toomey R, Kremen WS, Simpson JC, Samson JA, Seidman LJ, Lyons MJ, Faraone SV, Tsuang MT: Revisiting the factor structure for positive and negative symptoms: Evidence from a large heterogeneous group of psychiatric patients. Am J Psychiatry 1997;154:371-377.

18 Peralta V, Cuesta MJ: Dimensional structure of psychotic symptoms: An item-level analysis of SAPS and SANS symptoms in psychotic disorders. Schizophr Res 1999;38:13-26.

19 Costello CG: Research on symptoms versus research on syndromes. Arguments in favor of allocating more research time in the study of symptoms. Br J Psychiatry 1992;160:304308.

20 Liddle PF, Friston KJ, Frith CD, Hirsch SR, Jones T, Frackowiak RS: Patterns of cerebral blood flow in schizophrenia. Br J Psychiatry 1992;160:179-186.

21 Mauri M, Borri C, Giannotti D, Zambotto S, Cassano GB, Akiskal HS: Psychotic symptom patterns and the diagnosis of schizophrenia. Psychopathology 1992;25:5-10.

22 Aderibigbe YA, Gureje O: Symptom dimensions of schizophrenia: A selective integration of neurophysiological and neuropsychological correlates. Neuropsychobiology 1996;34:192200. chiatry Res 1999;90:159-168.
23 Berner P: Conceptualization of schizophrenia: The symptom-oriented approach. Psychopathology 1997;30:251-256.

24 Stuart GW, Pantelis C, Klimidis S, Minas IH: The three-syndrome model of schizophrenia: Meta-analysis of an artifact. Schizophr Res 1999;39:233-242.

25 Kay SR, Opler LA, Lindenmayer JP: The Positive and Negative Syndrome Scale (PANSS): Rationale and standardization. Br J Psychiatry 1989;7:59-67.

26 Andreasen NC, Olsen S: Negative vs. positive schizophrenia. Definition and validation. Arch Gen Psychiatry 1982;39:789-794.

27 Sabri O, Erkwoh R, Schreckenberger M, Owega A, Sass H, Buell U: Correlation of positive symptoms exclusively to hyperperfusion or hypoperfusion of cerebral cortex in never-treated schizophrenics. Lancet 1997;349:1735-1739.

-28 Nurnberger JI, Blehar MC, Kaufmann CA, York-Cooler C, Simpson SG, Harkavy-Friedman J, Severe JB, Malaspina D, Reich T: Diagnostic interview for genetic studies: Rationale, unique features, and training - NIMH Genetics Initiative. Arch Gen Psychiatry 1994; 51:849-859.

29 Raichle ME, MacLeod AM, Snyder AZ, Powers WJ, Gusnard DA, Shulma GL: A default mode of brain function. Proc Nat Acad Sci 2001;98:676-682.

30 Wicker B, Ruby P, Royet JP, Fonlupt P: A relation between rest and the self in the brain? Brain Res Rev 2003;43:224-230.

- 31 Kelley WM, Nacrea CN, Wyland CL, Caglar $\mathrm{S}$, Inati S, Heatherton TF: Finding the self? And event-related fMRI study. J Cogn Neurosci 2001;14:785-794.

> 32 Blackwood NJ, Bentall RP, Ffytche DH, Simmons A, Murray RM, Howard RJ: Persecutory delusions and the determination of self-relevance: An fMRI investigation. Psychol Med 2004;34:591-596.

33 Gallagher HL, Frith CD: Functional imaging of 'theory of mind' Trends Cogn Sci 2003; 7 : $77-83$.

34 Peralta V, Cuesta MJ: How many and which are the psychopathological dimensions in schizophrenia? Issues influencing their ascertainment. Schizophr Res 2001;49:269-285.

35 Garety PA, Freeman D: Cognitive approaches to delusions: A critical review of theories and evidence. Br J Clin Psychol 1999;38:113154 